Asthma due to lymphocytosis is a variety of asthma chiefly found in children, especially nurslings, and the blood count in all the cases $I$ have investigated has shown a decided lymphocytosis. In one case the blood count showed 93,500 white cells, 70 por cent. of which were lymphocytes, and a vast majority of these lymphocytes were small, showing that they had come directly from the lymph chanuels and the chyle. The reason for the dyspnea in this variety of asthma is twofold: 1. A sufficient amount of oxygen can not reach the lungs, owing to the narrowed respiratory tubes, including the pharynx, larynx, trachea and bronchi, due to the collateral engorgement of their Jymph channels. 2. The oxygen that finally reaches the lungs is handicapped in reaching the hemoglobin in the blood, owing to the hemoglobin being so thickly surrounded by lymphocytes.

Local treatment may do some good, but systemic treatment will do most good. The leucocytic variety of asthma $I$ have found chicfly in adults and it usually starts from an intestinal indigestion and toxemia which cause a toxic leucocytosis, and an enlargement of the glandular structures of the larynx and trachea.

The glandular enlargement first appears in the larynx and is usually most pronounced during an attack following a long period, perhaps months, of freedom and not infrequently this enlargement will manifest itself in the patient weeks before an attack through the snugness of his collar and change of voice.

When the attack first begins, the obstruction and wheezing is confined entirely to the larynx. After a few days the laryngeal glands undergo a revolution and softening and the laryngeal dyspnea disappears, with the expectoration of mouthfuls of thick, viscid mucus containing Curschmann's spirals, leucocytes and eosinophile cells. There has always been a doubt as to the origin of Curschmann's spirals. 'They always appear in the sputum first exuded in the leucocytic variety of asthma, whether this sputum comes from enlarged laryngeal or tracheal glands, but owing to the fact that they appear in the sputum coming from the enlarged laryngeal glands before the process has extended further down, proves conclusively that they do not in any case originate in the smallest bronchioles as their easts, as heretofore supposed. The anemic variety of asthma we find chiefly in the extremes of life. It comes on night after night simply from the lack of the action of sunlight on the blood. There is an individual peculiarity of the anemic asthmatic. The dyspnea is not alone due to the small amount of hemoglobin in the blood, but also to the fact that the hemoglobin does not readily absorb or carry oxygen, and this is in fact due to a deficiency in animal magnetism. The lack of the chemical effect of sunlight on the blood often proves to be the last straw. Altitude, by increasing the blood count, will in this variety effect an immediate cure.

Dr. Casselberry-I would like to know in which class the Doctor would place the asthma found in connection with hay fever?

DR. JACK-I think there is a close association between hay asthma and the leucocytic variety of asthma. I have in many cases found a leucocytosis in hay asthma, and I am of the opinion that pollen dust will, in susceptible cases, produce a leucocytosis sufficient to bring on an attack of asthma.

Dr. EMIL Ambers, Detroit-Blood counts have been made in cases of adenoids and it has been seen upon investigation that after an adenoid operation the conditions of the blood became more favorable. If merely the toxic effects in the blood were to account for it we should not expect this. If the symptoms appear to be due to mechanical obstruction and if the removal of the adenoids improves the symptoms, we may as well come to the conclusion that the mechanical obstruction furnishes the cause and that the changes in the composition of the blood must be regarded as the effect, and not vice versa.

DR. J. F. BARNhilL, Indianapolis-I should like to ask the gentleman, who had the floor before the last speaker, in what specialty he is practicing and from what field he has made his observation. I make that query because rhinologists have given long lists of cases in which they have cured asthma by operation alone. Some time ago I wrote to the professors of medicine in each regular college in the west, asking whether, in his experience, asthma had ever been cured by internal medicine alone, and there was in every instance a negative response.

DR. $J_{A C K}-\mathrm{I}$ am in general practice.

Dr. J. H. FarreLd, in closing-It is all very well to go into the discussion of the blood in the production of asthma, but facts are very stubborn things. In my experience, as well as in the experience of men of longer practice, cases of asthma are often cured by operation. When in general practice I saw many cases of asthma, but I never saw a case in which the attack could not be cut short by the hypodermic injection of $1 / 1 \mathrm{gr}$. of morphin, and 1-100 gr. of atropin. In Arizona I saw many cases, but I never saw a case cured by the change of climate, except such as we could attribute to the absorption of in tammatory conditions in the nasal chamber.

\section{AN UNUSUAL ANOMALY OF THE FAUCIAL TONSIL.*}

GEORGE L. RIUFARDS, M.D. FALL RIVER, MASS.

The patient was a woman, 60 years old, who reported that her throat had been paining her for the past two years, and that for a long time, almost as long as she could remember, she had had more or less discomfort in the region of each tonsil. The right was found to be somewhat hypertrophied, and she had pain running to the ear from this region. She did not at first want the tonsil removed, and was treated for some weeks with chromic acid applications. This was followed by some improvement in the sensation. Later she consented to the removal of the right tonsil; the left one was not large. On applying a tonsillotome I was surprised to find that I was unable to cut through the tonsil, and on examining with the finger found that a bony mass penetrated the tonsil almost to its outer border. This was removed with a stout bone-cutting forceps, and on being examined was found to be a portion of the styloid process, the center of the bone showing a distinct marrow. I examined the other side with the finger, and found that, though not so prominent, the tip of the process could be felt through the left tonsil as well.

Not having seen any literature in reference to anything of the kind, I wrote to Dr. Thomas Dwight, professor of anatomy in Harvard University, in regard to the matter, and received from him the following reply:

"Your case is a very interesting one. I do not remember of hearing of a styloid that touched the tonsil, and yet I have not the slightest doubt that you had to do with a styloid, and the explanation is simple enough. Long styloids are not uncommon; and remarkably long ones are due to the ossification of the stylohyoid ligament. On its way to the lesser horn of the hyoid it must pass very close to the tonsil. There is in the museum a skull in which this is ossified throughout, though, I think, with one interruption on each side between the skull and the hyoid. I saw to-day a temporal bone with a styloid, the point of which was nearly, two and one-half inches from the lower border of the external auditory meatus. This must have reached to the level of the tonsil.

"It is perfectly in accord with all we know of the process of growth of organs, that if a bony structure should encroach on the domain of adenoid tissue the latter should, so to speak, fold itself around it."

* Read in the Section on Laryngology and Otology, at the Fiftysecond Annual Meeting of the American Medical Association, beld at St. Paul, Minn., June 4-7, 1901. 
If any member of this Section, or any reader, has seen anything similar, I would esteem it a favor if he would inform me as to the circumstances of the case.

\section{THE EFFECT WHICH THE SO-CALLED CATAR- RHAL DISEASES OF THE NOSE AND THROAT MAY HAVE UPON THE GENERAL HEALTH.* \\ CAROLUS M. COBB, M.D. BOSTON, MASS.}

\begin{abstract}
Myself when young did eagerly frequent Doctor and saint, and heard great argument About it, and about; but evermore

Came out by the same door wherein I went.
\end{abstract}

Thus sings that old cynic Omar Khayyam, and Omar's criticism of theologic discussions applies with more or less force to medicine. It is so much easier to accept an explanation already made, than to think about the reason for ourselves, that what argument we hold is likely to be about the details or variations of already existing theories, without much inquiry whether they may be right or wrong. The explanation of any local disease which we do not thoroughly understand, and therefore fail to treat successfully, is simplicity itself. We say, with many variations of the wording perhaps, that it depends on the state of the general health. This explanation is very satisfactory to the patients and their friends, and it saves the physician the labor of finding the true cause for the condition. We are rarely very definite in our statements as to what the condition of the general health may be, and when we do come down to particulars, we are quite likely to think that the patient has the then fashionable disease. Fashions change in disease as they do in dress; just now the uric acid diathesis is a prime favorite; a few years ago it was congestion of the portal circulation.

In the treatment of disease of the nose and throat, we constantly meet with the explanation that the local disease depends on some disturbance of the general health. This has been so often and so confidently asserted, and so generally accepted, that it may seem too radical a statement to make. that the diseases of the nose and throat are not often caused by general conditions, and, further, that they are often the cause of the very state of the general health, which has been supposed to produce them. The exceptions to this statement are, syphilis, tuberculosis, rarefying osteitis, and some of the rarer forms of disease. The acute exanthematous diseases, as well as pneumonia and influenza, have not been considered because the involvement of the nose and throat is a part of the general infection. Certain conditions, as anemia and lowered vitality from whatever cause render the tissues more vulnerable, and other conditions which produce congestion, as indigestion, constipation, menstruation, may and often do cause an exacerbation of an already existing disease. This statement of the position which the state of the general health occupies as a cause of local disease, is perfectly consistent with the idea of the bacterial origin of the acute diseases of the nose and throat, and we have every reason to consider most of the so-called catarrhal diseases the sequelæ of the acute attacks. If we believe. as all progressive physicians to-day do, that most acute and many chronic diseases depend on germ infection, then the relation of the diseased conditions

* Read in the Section on raryngology and Otology, at the Fiftysecond Annual Metting of the American Medical Association, beld at st. Paul, Minn., June 4-7, 1901. of the nose and throat becomes of the utmost importance.

Assuming the infectious origin to be accepted, the question then is: In what way do the bacteria gain entrance to the system? The principal ways in which this takes place are through wounds, or through the respiratory, digestive, or genito-urinary tracts. Of these different routes of infection, we will consider only the respiratory. During normal respiration through the natural passages, Nature has thrown up a barrier, which not only prevents the entrance of the bacteria to the deeper parts of the respiratory tract, but warms and moistens the inspired air as well. When this barrier is rendered inoperative bv disease, or by nasal obstruction, the throat and lungs are exposed to the danger of infection. If, in addition to this loss of function of the nasal mucous membrane, there is a constant discharge of mucus or pus into the pharynx, the probability that the general health will be affected is very much greater. The effect that diseases of the nose and throat have upon the surrounding parts, as the ears, eyes, larynx and bronchial mucous membrane, and as a cause of headache, is a well-recognized clinical fact. If we attempt to analyze the different ways in which disease of the nose and throat may affect the general health, the subject naturally divides itself into two parts,1, the effect that may be produced by obstructed nasal respiration, and 2 , the effects produced by the migration of bacteria or by the absorption of the products of their action.

Under the first division we find those effects which are produced by mouth-breathing, such as anemia from lack of oxygen, disturbances of sleep, aprosexia, maldevelopment of the face if the mouth-breathing is established in early life, and frequent attacks of inflammation of the oropharynx, the tonsils, the larynx, and the bronchial mucous membrane. The effect upon the nervous system, of nasal obstruction. is marked, and varies in different cases through a wide range of symptoms, the most prominent of which are mental dulness, loss of memory, irritability and chorea. The effects produced by mouth-breathing are too common and have been too often reported to need more than a passing mention.

The second division needs more extended notice, because, while the effects may be no more serious, the connection between the local disease and the general condition is more likely to be overlooked. The different ways in which a nasal or nasopharyngeal discharge may affect the general health are: 1, the extension of the inflammation on the surface of the mucous membrane, either to the skin, where it causes excoriations of the skin, or more severe inflammation, as erysipelas, acne, etc., or to the larynx and bronchi, causing in this way protracted attacks of laryngitis and bronchitis. There is no doubt that this extension of the disease occurs, and it is probable that most of the cases of chronic laryngitis not caused by mouth-breathing, depend on disease higher up in the respiratory tract. The bronchial mucous membrane is involved in a large percentage of these cases and, where the bronchitis has this origin, the treatment is unsatisfactory unless the disease of the nose and throat is treated also. An illustration of the failure of treatment directed to the general health, and to the bronchial mucous membrane only, is seen in those cases of winter cough which finally run on to the so-called twenty years' consumption. These patients have usually taken, literally, barrels of $\mathrm{pv}^{-}$ pectorants and emulsions, and very few of them lave 